CrossMark \& click for updates

Cite this: Phys. Chem. Chem. Phys., 2015, 17, 31530

Received 27th February 2015 Accepted 15th June 2015

DOI: $10.1039 / c 5 c p 01183 c$

www.rsc.org/pccp

\section{Singular analysis and coupled cluster theory}

\author{
Heinz-Jürgen Flad, ${ }^{* a}$ Gohar Harutyunyan ${ }^{b}$ and Bert-Wolfgang Schulze ${ }^{c}$
}

\begin{abstract}
The primary motivation for systematic bases in first principles electronic structure simulations is to derive physical and chemical properties of molecules and solids with predetermined accuracy. This requires a detailed understanding of the asymptotic behaviour of many-particle Coulomb systems near coalescence points of particles. Singular analysis provides a convenient framework to study the asymptotic behaviour of wavefunctions near these singularities. In the present work, we want to introduce the mathematical framework of singular analysis and discuss a novel asymptotic parametrix construction for Hamiltonians of many-particle Coulomb systems. This corresponds to the construction of an approximate inverse of a Hamiltonian operator with remainder given by a so-called Green operator. The Green operator encodes essential asymptotic information and we present as our main result an explicit asymptotic formula for this operator. First applications to many-particle models in quantum chemistry are presented in order to demonstrate the feasibility of our approach. The focus is on the asymptotic behaviour of ladder diagrams, which provide the dominant contribution to shortrange correlation in coupled cluster theory. Furthermore, we discuss possible consequences of our asymptotic analysis with respect to adaptive wavelet approximation.
\end{abstract}

\section{Introduction}

Schrödinger's equation for many-particle systems interacting via Coulomb potentials provides a common starting point for computational models within the realm of $a b$ initio electronic structure theory. Recently, this field attracted considerable interest from applied mathematics, in particular numerical analysis. Let us just mention in this field, the work of Yserentant and coworkers on the mixed Sobolev regularity of Schrödinger's equation $^{1-5}$ and related work by others. ${ }^{6-8}$ Despite their tremendous significance for numerical simulations, ab initio post Hartree-Fock models, like coupled cluster (CC) theory, $c f^{9,10}$ and references therein. are rarely considered in the mathematical literature. Only recently, a first rigorous mathematical analysis of CC models has been undertaken by Schneider and Rohwedder. ${ }^{11-13}$

It is obvious from the point of view of numerical analysis, that apparent singularities of Coulomb potentials at coalescence points of particles limit any appropriate notion of regularity and represent a major bottleneck for $a b$ initio computer simulations. Therefore it is of great interest to get a detailed picture of the asymptotic behaviour of solutions to Schrödinger's equation

\footnotetext{
${ }^{a}$ Zentrum Mathematik, Technische Universität München, Boltzmannstr. 3, D-85747 Garching, Germany.E-mail: flad@ma.tum.de

${ }^{b}$ Institut für Mathematik, Technische Universität Berlin, Straße des 17. Juni 136, D-10623 Berlin, Germany

${ }^{c}$ Institut für Mathematik, Universität Potsdam, Am Neuen Palais 10,

D-14469 Potsdam, Germany
}

and related ab initio models, near coalescence points of particles. Following the pioneering work by Kato, ${ }^{14}$ M. \& T. HoffmannOstenhof and coworkers provided a fairly deep mathematical analysis for Schrödinger's equation. ${ }^{15-19}$ To the best of our knowledge, however, no rigorous attempt has been devoted to study the asymptotic behaviour of approximate ab initio models, like coupled (independent) electron pair approximations (C(I)EPA) or truncated models like coupled cluster singles and doubles (CCSD) in CC theory. It is the purpose of our work to develop mathematical techniques which enable a rather detailed insight into the asymptotic behaviour of these models and to derive actual consequences for computer simulations. For this, we first explored possible connections of our problem with the abstract mathematical framework of singular analysis, a branch of mathematics which studies the properties of functions, operators etc. in the presence of singularities. A particular pseudo-differential operator calculus, $c f$. the monographs, ${ }^{20-22}$ drew our attention because it provides the desired asymptotic information and reflects the hierarchical structure of our singularities in a natural manner. In the following we want to present a highly informal discussion of some basic concepts and essential features of this calculus, avoiding intricate notation, sophisticated mathematical arguments as well as lengthy calculations. Instead we discuss some first concrete applications which demonstrate the capabilities of our approach. There are other approaches in singular analysis which have been applied to electronic structure theory as well, here we have to mention e.g. the work of Mazzeo, Nistor and collaborators. ${ }^{23-25}$ 


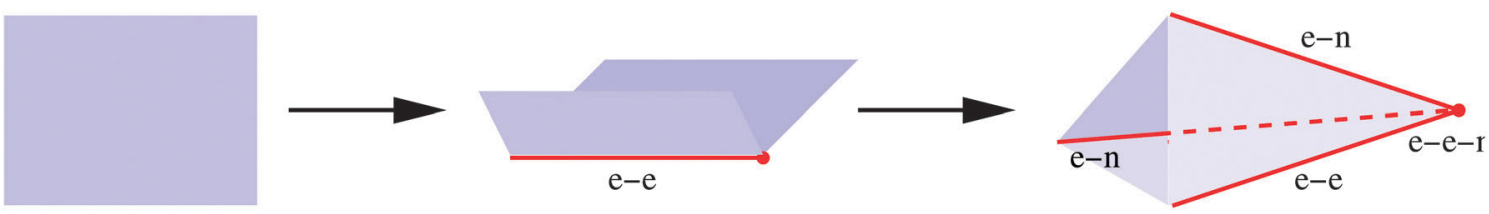

Fig. 1 Schematic illustration of the embedding scheme for a system consisting of two electrons and a nucleus. Once folding a sheet of paper represents the coalescence points of two electrons. Two more wrinkles correspond to coalescence points of electrons and the nucleus. All three wrinkles merge in a single point where both electrons are located at the nucleus.

\section{First encounter with singular analysis}

The mathematical notion of a singularity of a function refers to a point where the function is not defined or in a particular sense not well-behaved. Its meaning is therefore more general as in physics where it usually refers to points where the function becomes infinite. Vice versa one can specify singularities of a geometrical object by means of appropriate characteristic functions. Examples are metric or curvature tensors defined on a manifold, which might become degenerate or divergent at singularities. Another example is the dimensionality of tangent space considered as a function on an algebraic variety. Singularities may appear as isolated points or accumulate to lower dimensional subspaces, imposing a characteristic geometrical structure on the space which contains the domain of the function. Beyond that, singular analysis deals with function spaces defined on geometrical objects, like manifolds, with singularities and operators acting on them. Here again, the singular structure is represented by special weights in the definition of function spaces or by operators which become degenerate in the neighbourhood of a singularity. In order to illustrate these concepts let us consider the wavefunction of a single electron defined on its configuration space $\mathbb{R}^{3}$. In Cartesian coordinates, the quadratic differential form of the metric tensor

$$
\mathrm{d} s^{2}=\mathrm{d} x^{2}+\mathrm{d} y^{2}+\mathrm{d} z^{2}
$$

is everywhere non degenerate. Instead taking spherical coordinates, the quadratic differential form of the metric tensor

$$
\mathrm{d} s^{2}=\mathrm{d} r^{2}+r^{2}\left[\mathrm{~d}^{2} \theta+\sin ^{2} \theta \mathrm{d} \phi^{2}\right]
$$

becomes degenerate along the $z$-axes and in particular at the origin. Obviously it is an artifact of this particular coordinate system without any physical relevance. However, if we consider the electron in a singular Coulomb potential of a point charge centered at the origin, i.e., a hydrogen atom, the degeneracy of the metric tensor perfectly reflects the singular behaviour of the wavefunction, which is smooth except at the origin. This approach can be generalized to many-electron systems, interacting with each other and fixed nuclei via singular Coulomb potentials. It is well known, that many-electron wavefunctions are smooth except at coalescence points of electrons ${ }^{14}$ or where electrons approach a nucleus. The basic idea is to find an appropriate coordinate system whose metric tensor reflects the singular structure of configuration space. The number of electrons which join at a coalescence point imposes a natural hierarchy on these singularities. Two electrons approaching each other or an electron approaching a nucleus will be considered as an edge singularity in configuration space as long as the pair stays away from the other particles. A corner singularity in the configuration space appears if such a colliding pair approaches another electron or nucleus. Along these lines it is possible to define a sequence of higher order singularities which pervade configuration space. We refer to this construction as an embedding scheme for coalescence points of particles into configuration space. By simply folding a sheet of paper, we give in Fig. 1 an illustration of this embedding scheme for a system consisting of two electrons and a nucleus. This simple picture exemplifies our notion of edge and corner singularities in configuration space.

\subsection{Stratified spaces and degenerate operators}

In mathematics such an embedding scheme corresponds to a stratified space ${ }^{26,27}$ where strata are classified according to the number of merging particles. To be more precise, let us consider a Coulomb system consisting of $N$ electrons and several nuclei in the Born-Oppenheimer approximation where nuclei are kept fixed and the configuration space restricts to electronic degrees of freedom. First of all, the physical configuration space $\mathcal{M}$ of $N$ electrons $\dagger$ can be identified with $\mathbb{R}^{3 N}$. Let us define the subset $\mathcal{M}_{0} \subset \mathcal{M}$ of all possible coalescence points of particles including any number of electrons and nuclei. With it, $\mathcal{M} \backslash \mathcal{M}_{0}$ can be considered as an open smooth manifold $\ddagger$ or more general as the inner part of an open smooth manifold with boundary. Next let us consider the subset $\mathcal{M}_{1} \subset \mathcal{M}_{0}$ of all coalescence points of more than two particles. The stratum $\mathcal{M}_{0} \backslash \mathcal{M}_{1}$ is an open smooth manifold representing edges of $\mathcal{M}$. Correspondingly, we denote $\mathcal{M} \backslash \mathcal{M}_{1}$ as a singular manifold with edges. Higher order strata can be constructed along the same lines, e.g., let the subset $\mathcal{M}_{2} \subset \mathcal{M}_{1}$ denote the set of coalescence points of more than three particles. Again the stratum $\mathcal{M}_{1} \backslash \mathcal{M}_{2}$ is an open smooth manifold representing the lowest order type of corners in $\mathcal{M}$. Therefore $\mathcal{M} \backslash \mathcal{M}_{2}$ is a singular manifold with edges and corners. In this way the configuration space can be decomposed into its strata, i.e.,

$$
\mathcal{M}=\mathcal{M} \backslash \mathcal{M}_{0} \cup \mathcal{M}_{0} \backslash \mathcal{M}_{1} \cup \mathcal{M}_{1} \backslash \mathcal{M}_{2} \ldots
$$

In Fig. 2, we illustrate this stratification process by cutting the three times folded sheet of paper from Fig. 1 into its strata and

† We do not consider spin degrees of freedom or equivalent permutational symmetries of the electron coordinates in our discussion.

\$ The manifold $\mathcal{M} \backslash \mathcal{M}_{0}$ actually corresponds to the mathematical notion of a configuration space of $N$ ordered particles in $\mathbb{R}^{3}$. 


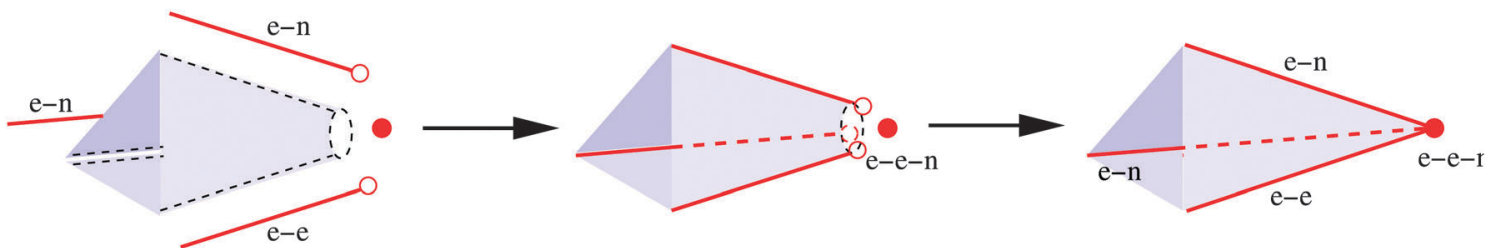

Fig. 2 Stratification of the singular configuration space of two electrons and a nucleus depicted in Fig. 1. Removing all coalescence points of particles yields the open smooth manifold $\mathcal{M} \backslash \mathcal{M}_{0}$ and various strata. Reconstruction of the singular manifold by first adding the edge strata gives $\mathcal{M} \backslash \mathcal{M}_{1}$ and finally $\mathcal{M}$ after adding the corner singularity $\mathcal{M}_{1}$.

do a reconstruction by adding successively singularities of increasing order.

In order to do any meaningful analysis on stratified spaces it is necessary to demand existence of a control function for each stratum. This function is defined in an appropriate tubular neighbourhood of the stratum and provides on the neigbouring strata of higher codimension a measure of the distance to the stratum. A control function $g$ of a stratum $S$ has to be positive semidefinite, with nonvanishing gradient and $S=\{x \in M: g(x)=0\}$, cf. ref. 27. In our simple example, depicted in Fig. 3, control functions $r$ and $t$ are associated with edge and corner strata, respectively.

The next step is to define appropriate differential operators on a singular manifold $\mathcal{M}$. These differential operators are defined on $\mathcal{M}_{0}$ and possess certain characteristic type of degenerate behaviour near lower dimensional strata. In order to make this degenerate behaviour more precise, let us consider some generic model spaces which locally represent the nonsingular part of configuration space $\mathcal{M}_{0}$ in a neighbourhood of a stratum.

The simplest case is a conical singularity, where the stratum corresponds to a single point. This happens, e.g., at the location of a nucleus in the configuration space of a single electron. Near a conical singularity, $\mathcal{M}_{0}$ can be represented by an open stretched cone: $X^{\wedge}:=\mathbb{R}_{+} \times X$ with base $X$. In our specific example, $X$ is given by the two sphere $S^{2}$. On the stretched cone $X^{\wedge}$ let us define a class of degenerate $m^{\prime}$ th order differential operators $\operatorname{Diff} m$ deg $\left(X^{\wedge}\right)$ which are of the form

$$
A=r^{-m} \sum_{j \leq m} a_{j \alpha}(r)\left(-r \partial_{r}\right)^{j} .
$$

Here coefficients $a_{j \alpha}(r)$ represent $m-j$ 'th order differential operators on the base $X$ and are smooth up to $r=0$. In the following, this class of differential operators is denoted by $\operatorname{Diff}^{m-j}(X)$. A simple example where these conditions are obviously satisfied is the shifted Hamiltonian of the hydrogen atom in spherical polar coordinates

$$
H-E=-\frac{1}{r^{2}}\left[\frac{1}{2}\left(-r \frac{\partial}{\partial r}\right)^{2}-\frac{1}{2}\left(-r \frac{\partial}{\partial r}\right)+\frac{1}{2} \Delta_{S^{2}}+r Z+r^{2} E\right],
$$

which belongs to the operator class $\operatorname{Diff} f_{\operatorname{deg}}^{2}\left(X^{\wedge}\right)$. The next higher singularities, we have to deal with are of edge type and correspond to coalescence points of two particles. Locally $\mathcal{M}_{0}$ can be represented by an open stretched edge (wedge): $\mathbb{W}:=X^{\wedge} \times Y, Y$ is an open subset of $\mathbb{R}^{n}$ where $n$ depends on the dimension of the edge. On a wedge let us define a class of edge degenerate $m^{\prime}$ th order differential operators Diff deg $^{m}(W)$ which are of the form

$$
A=r^{-m} \sum_{j+|\alpha| \leq m} a_{j \alpha}(r, y)\left(-r \partial_{r}\right)^{j}\left(r D_{y}\right)^{\alpha}
$$

with coefficients $a_{j \alpha}(r, y) \in \operatorname{Diff}^{m-j-|\alpha|}(X)$ smooth in $r, y$ up to $r=0$.

Finally, we consider corner type singularities which correspond to coalescence points of three particles. Near such a corner, $\mathcal{M}_{0}$ can be locally represented by an open stretched corner $\mathbb{M}=\mathbb{R}_{+} \times X^{\wedge} \times Y$. The class of corner degenerate $m^{\prime}$ th order differential operators requires two independent distance parameters $r$ and $t$. Like before $r$ controls the distance to the nearest edge stratum and $t$ controls the distance to the corner stratum itself. The class Diff $f_{\text {deg }}^{m}(\mathbb{M})$ consists of all $m^{\prime}$ th order differential operators of the form

$$
A=t^{-m} r^{-m} \sum_{k+j+|\alpha| \leq m} a_{k j \alpha}(t, r, y)\left(-r \partial_{r}\right)^{j}\left(r D_{y}\right)^{\alpha}\left(-r t \partial_{t}\right)^{k}
$$

with coefficients $a_{k j \alpha}(t, r, y) \in \operatorname{Diff}^{m-j-|\alpha|}(X)$ smooth up to $r, t=0$.

\subsection{The Hamiltonian as an edge/corner degenerate operator}

In the previous section, a general ansatz for degenerate differential operators on conical, edge and corner spaces has been given.

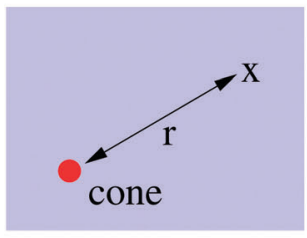

a)

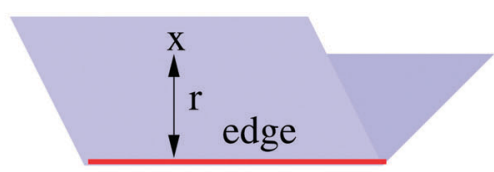

b)

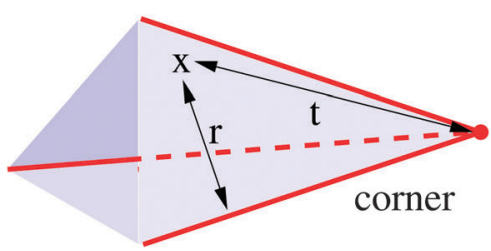

c)

Fig. 3 Control functions associated to the strata of singular configuration spaces of one and two electrons and a nucleus. (a) One electron case: $r$ controls the distance to the conical singularity. $(b, c)$ Two electron case: $r$ and $t$ control the distance to edge and corner strata. 
It is the purpose of this section to show that the Hamiltonian of a many-particle Coulomb system, represented in appropriate coordinates, actually fits into this scheme. Our focus will be on two-electron Hamiltonians, it is however possible to extend the following considerations to any number of electrons. First of all, we want to discuss the helium atom, a paradigm for electronpair correlation, but also effective Hamiltonians, derived from coupled or independent electron-pair models, are considered. The Hamiltonian of the helium atom, here given in Cartesian coordinates,

$$
H=-\frac{1}{2}\left(\Delta_{1}+\Delta_{2}\right)-\frac{2}{\left|x_{1}\right|}-\frac{2}{\left|x_{2}\right|}+\frac{1}{\left|x_{1}-x_{2}\right|},
$$

has been the subject of numerous analytical studies ${ }^{28-31}$ and represents one of the favourite benchmark problems for numerical methods aiming for very high accuracies. ${ }^{32}$

Various coordinate systems have been discussed in the literature in order to represent the singular structure of the helium wavefunction, among them are Fock's hyperspherical coordinates $^{33-36}$ and Hylleraas coordinates ${ }^{37-40}$ which have been widely used for numerical simulations of two-electron systems. Although these coordinate systems contain appropriate coordinates to control the distance to singular strata, the corresponding Hamiltonians, however, fail to satisfy the crucial requirement having coefficients which are smooth up to the stratum. Nevertheless, at least one particular coordinate system exists, see e.g. ${ }^{41,42}$ which satisfies all of our requirements. It represents a special kind of hyperspherical coordinates where $\mathbb{R}^{6}$ corresponds to a conical manifold $\left(\overline{\mathbb{R}}_{+} \times S^{5}\right) /\left(\{0\} \times S^{5}\right)$ with embedded edge singularities $Y_{1}, Y_{2}, Y_{3}$ on the hypersphere $S^{5}$, see Fig. 4. Explicitly, the edges $Y_{1}, Y_{2}$ represent coalescence points of an electron and the nucleus, whereas $Y_{3}$, corresponds to coalescence points of the two electrons. The embedded two dimensional edges $Y_{i}, i=1,2,3$, themselves are homeomorphic to the two sphere $S^{2}$. A detailed mathematical discussion of this coordinate system and its generalization to $N$ electrons is presented in ref. 43. Because of its significance and in order to make the paper reasonably self contained, we present some particularly relevant features of these coordinates in some detail below, $c f$. also our related discussion in ref. 44 .

Explicit relations to Cartesian coordinates in $\mathbb{R}^{6}$ are given by

$$
\begin{gathered}
x_{1}=t \sin r \sin \theta_{1} \cos \phi_{1}, \quad x_{2}=t \sin r \sin \theta_{1} \sin \phi_{1}, \\
x_{3}=t \sin r \cos \theta_{1}, \\
x_{4}=t \cos r \sin \theta_{2} \cos \phi_{2}, \quad x_{5}=t \cos r \sin \theta_{2} \sin \phi_{2}, \\
x_{6}=t \cos r \cos \theta_{2},
\end{gathered}
$$

with hyperspherical radius

$$
t:=\sqrt{x_{1}^{2}+x_{2}^{2}+x_{3}^{2}+x_{4}^{2}+x_{5}^{2}+x_{6}^{2}},
$$

which controls the distance to the corner singularity and radial variable $0<r \leq \pi / 2$, where radial "distances" $r=0, r=\pi / 4$, $r=\pi / 2$ correspond to $e_{1}-n, e_{1}-e_{2}, e_{2}-n$ edge singularities, respectively. The remaining variables $\left(\theta_{1}, \phi_{1}\right)$ and $\left(\theta_{2}, \phi_{2}\right)$ are spherical variables on $X$ and $Y_{i}$, respectively. Instead of a single global coordinate system, it is more convenient to take an atlas of three local coordinate systems, each assigned to a particular edge, such that the radial variable $r$ vanishes for this edge. In the case of the $e-e$ edge it is therefore convenient to define hypersherical coordinates with respect to center of mass coordinates

$$
\begin{aligned}
z_{i} & =\frac{1}{\sqrt{2}}\left(x_{i}-x_{i+3}\right), \\
z_{i+3} & =\frac{1}{\sqrt{2}}\left(x_{i}+x_{i+3}\right) \quad \text { for } i=1,2,3 .
\end{aligned}
$$

In order to comprehend the singular structure imposed by these coordinates, it is helpful to consider the corresponding quadratic differential form of the metric tensor

$$
\begin{aligned}
\mathrm{d} s^{2}=\mathrm{d} t^{2} & +t^{2}\left[\mathrm{~d} r^{2}+\sin ^{2} r\left(\mathrm{~d} \theta_{1}{ }^{2}+\sin ^{2} \theta_{1} \mathrm{~d} \phi_{1}{ }^{2}\right)\right. \\
& \left.+\cos ^{2} r_{1}\left(\mathrm{~d} \theta_{2}{ }^{2}+\sin ^{2} \theta_{2} \mathrm{~d}{\phi_{2}}^{2}\right)\right]
\end{aligned}
$$

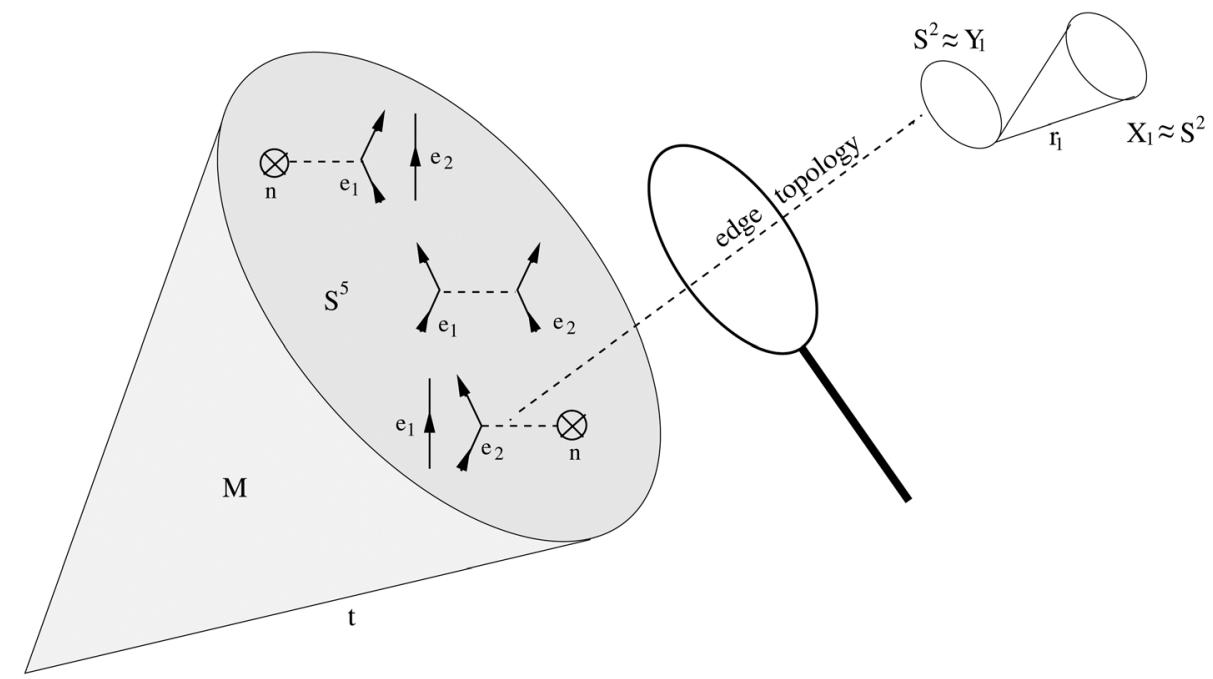

Fig. 4 Schematic representation of hypershperical coordinates for the helium atom. On the five sphere $S^{5}$, embedded two dimensional edges are symbolized by Feynman diagrams. 
It can be seen that the metric is degenerate with respect to both distance variables $r$ and $t$. The part in square brackets represents the metric on the five sphere $S^{5}$ and becomes degenerate at the corner singularity. On $S^{5}$, the metric becomes degenerate at the edge to which the coordinates refer.

With respect these coordinates, the Hamiltonian of the helium atom becomes edge degenerate

$$
H=r^{-2}\left(\sum_{j+|\alpha| \leq 2} a_{j \alpha}(r, y)\left(-r \frac{\partial}{\partial r}\right)^{j}\left(r D_{y}\right)^{\alpha}+\frac{r}{t} v_{e-e(n)}\right),
$$

with coefficients $a_{j, k, \alpha} \in C^{\infty}\left(\overline{\mathbb{R}}_{+} \times Y_{i}, \operatorname{Diff}^{2-j-|\alpha|}(X)\right)$ and eventually corner degenerate

$$
\begin{aligned}
H= & t^{-2} r^{-2} \\
& \times\left(\sum_{j+k+|\alpha| \leq 2} a_{j, k, \alpha}(t, r, y)\left(-r t \frac{\partial}{\partial t}\right)^{j}\left(-r \frac{\partial}{\partial r}\right)^{k}\left(r D_{y}\right)^{\alpha}+r t v_{e-e(n)}\right)
\end{aligned}
$$

with coefficients $a_{j, k, \alpha} \in C^{\infty}\left(\overline{\mathbb{R}}_{+} \times \overline{\mathbb{R}}_{+} \times Y_{i}\right.$, $\left.\operatorname{Diff}^{2-j-|\alpha|}(X)\right)$. The potential functions $v_{e-e(n)}$ belong to $C^{\infty}\left(\overline{\mathbb{R}}_{+} \times S_{1}^{2} \times S_{2}^{2}\right)$ with explicit expressions in the corresponding coordinates given by

$$
\begin{aligned}
v_{e-n}\left(r, \theta_{1}, \phi_{1}, \theta_{2}, \phi_{2}\right):= & -\frac{Z r}{\sin r}-\frac{Z r}{\cos r} \\
& +\frac{r}{\sqrt{1-\sin (2 r) a\left(\theta_{1}, \phi_{1}, \theta_{2}, \phi_{2}\right)}}
\end{aligned}
$$

and

$$
\begin{aligned}
v_{e-e}\left(r, \theta_{1}, \phi_{1}, \theta_{2}, \phi_{2}\right):= & -\frac{\sqrt{2} Z r}{\sqrt{1+\sin (2 r) a\left(\theta_{1}, \phi_{1}, \theta_{2}, \phi_{2}\right)}} \\
& -\frac{\sqrt{2} Z r}{\sqrt{1-\sin (2 r) a\left(\theta_{1}, \phi_{1}, \theta_{2}, \phi_{2}\right)}} \\
& +\frac{r}{\sqrt{2} \sin r},
\end{aligned}
$$

respectively. Here, the angular dependence is represented by the function

$$
a\left(\theta_{1}, \phi_{1}, \theta_{2}, \phi_{2}\right):=\cos \theta_{1} \cos \theta_{2}+\sin \theta_{1} \sin \theta_{2} \cos \left(\phi_{1}-\phi_{2}\right) .
$$

\subsection{Algebra of pseudo-differential operators on stratified spaces}

In the previous sections we have shown that the configuration space and Hamiltonian of an interacting Coulomb system matches the requirements imposed by an abstract theory of singular analysis. Once this has been established, the whole machinery of this sophisticated theory is at our disposal $c f$. ref. 20-22. It is the purpose of this section to give a rather informal account of the most significant gains.

In the following, we restrict our discussion to Hamiltonian operators for electron-pairs, interacting via a Coulomb potential, with external potentials which are either of Coulomb type or represent effective local potentials due to the presence of other electrons not explicitly taken into account. In particular it will be assumed that the exchange part is local, e.g., given in terms of an optimized effective potential. Concerning these effective potentials, we will always assume that the required regularity properties are satisfied. A rigorous proof for Hartree potentials has been given in ref. 45 .

The general approach to extract asymptotic information is based on the concept of a parametrix of the shifted Hamiltonian operator $A(\lambda):=H-\lambda$ where $\lambda \in \mathbb{R}$ might be an eigenvalue. In a certain sense, a parametrix can be considered as an approximate inverse of a Hamiltonian. To make this concept more precise, let us suppose that the shifted Hamiltonian and its parametrix are bounded operators between appropriate function spaces, i.e.,

$$
A: \mathcal{F}_{1} \rightarrow \mathcal{F}_{2}, \quad P: \mathcal{F}_{2} \rightarrow \mathcal{F}_{1}
$$

According to its definition, a parametrix satisfies the equations

$$
P A=I+G_{1} \text { and } A P=I+G_{\mathrm{r}},
$$

i.e., $P$ is the left(right) inverse of $A$ modulo the Green operators $G_{\mathrm{l}}\left(G_{\mathrm{r}}\right)$. Here the basic idea is that the Green operator $G_{\mathrm{l}}\left(G_{\mathrm{r}}\right)$ maps $\mathcal{F}_{1}\left(\mathcal{F}_{2}\right)$ into a particularly nice subspace $S_{1} \subset \mathcal{F}_{1}\left(S_{2} \subset \mathcal{F}_{2}\right)$. It is clear from the definition, that a parametrix of a differential operator in $\operatorname{Diff}_{\mathrm{deg}}^{m}(\mathcal{M})$ must belong to a wider class of operators, which are the so-called pseudo-differential operators. These operators are grouped into classes $L^{S}(\mathcal{M})$, with $s \in \mathbb{R}$, such that $\operatorname{Diff}_{\text {deg }}^{m}(\mathcal{M}) \subset L^{m}(\mathcal{M})$, with $m \in \mathbb{N}$, and a parametrix $P$ for $A \in$ $\operatorname{Diff}_{\text {deg }}^{m}(\mathcal{M})$ belongs to $L^{-m}(\mathcal{M})$. For further reference, let us denote the whole class of Green operators by $L_{G}(\mathcal{M})$. In order to guarantee the existence of a parametrix, the corresponding differential operator must be elliptic in an appropriate sense. A general discussion of the concept of ellipticity has been given in the monograph ${ }^{22}$ and a detailed proof of ellipticity for Hamiltonian operators in ref. 44.

In the classical pseudo-differential operator calculus, the nice subspace $S$ corresponds to the space of smooth functions. However, due to the singular nature of our problem this cannot be the case here, instead Green operators map into function spaces with specific asymptotic behaviour. Let us consider e.g. an edge singularity, where the space $S_{1} \subset \mathcal{F}_{1}$ can be written as a direct sum

$$
S_{1}=\mathcal{E}_{1}^{N} \oplus \mathcal{F}_{1}^{N}
$$

with asymptotic subspace

$$
\mathcal{E}_{1}^{N}:=\operatorname{span}\left\{\omega(r) \sum_{j} \sum_{k=0}^{m_{j}} c_{j k}\left(\phi_{1}, \theta_{1}\right) v_{j k}\left(t, \phi_{2}, \theta_{2}\right) r^{-p_{j}} \ln ^{k} r\right\}
$$

and flattened space $\mathcal{F}_{1}^{N}$. Here, $\omega$ denotes an appropriate cut-off function for $r \gg 0$ and the asymptotic behaviour near the edge 
is characterized by a finite number of discrete parameters $p_{j}$ which are located in a strip of the complex plane, i.e.,

$$
p_{j} \in\left\{\frac{3}{2}-\gamma-N<\Re z<\frac{3}{2}-\gamma\right\},
$$

with weight $\gamma$ depending on the specific application, $c f$. ref. 44 for further details. In this expansion, asymptotic coefficients correspond to smooth functions $c_{j k}$ and $v_{j k}$ on the basis of the cone and edge stratum, respectively. The flattened space $\mathcal{F}_{1}^{N}$ consists of functions $u$ such that $r^{-N+\varepsilon} u$ still belongs to $\mathcal{F}_{1}$ for any $\varepsilon>0$. Operators which belong to a class $L^{S}(\mathcal{M})$ provide maps between asymptotic subspaces, i.e., they preserve asymptotic information.

With our particular application in mind, it might seem unnecessary complicated to consider asymptotic subspaces (2.21) with complex exponents $p_{j}$. In the pseudo-differential calculus, however, the values of the exponents have to be calculated and actually correspond to poles of meromorphic functions. Therefore it is rather natural to assume complex exponents in the general setting. In this respect our approach differs from the Fock expansion where a specific asymptotic behaviour, i.e., integer exponents, has been assumed from the very beginning. We want to emphasize, that any ad hoc ansatz for an expansion requires a proof, that it actually converges to a solution. A question which is still not fully settled in the case of the Fock expansion, $c f$. ref. 28-31.

With this background information at hand, it is easy to see how the parametrix and associated Green operator provides a tool to study the asymptotic behaviour of solutions of linear equations of type

$$
A(\lambda) u=f
$$

where $f$ itself has a well defined asymptotic behaviour. Let us first consider an eigenvalue problem $A(\lambda) u=0$ where $\lambda$ corresponds to an eigenvalue. Application of the parametrix from the left yields

$$
P(\lambda) A(\lambda) u=\left(I+G_{1}(\lambda)\right) u=0, \quad \rightarrow \quad u=-G_{1}(\lambda) u,(2.24)
$$

which means that the asymptotic behaviour of an eigenfunction is completely determined by the left Green operator $G_{1}$. Similarly, for any solution $u$ of the linear eqn (2.23) one gets

$$
P(\lambda) A(\lambda) u=\left(I+G_{1}(\lambda)\right) u=P(\lambda) f \quad \rightarrow \quad u=P(\lambda) f-G_{1}(\lambda) u,
$$

which means that the parametrix and associated Green operator determine its asymptotic behaviour.

\subsection{Asymptotic parametrices for Hamiltonian operators}

The construction of a parametrix for a Hamiltonian operator and its corresponding Green operator can be done in a systematic manner. Within the present work, the whole purpose of this procedure is to get asymptotic properties of wavefunctions near singularities. This can be most easily achieved by introducing the concept of an asymptotic parametrix which can be evaluated order by order via a recurrence scheme. Let us first note that every operator $O \in L^{S}(\mathcal{M})$ can be written in the form

$$
O=\sum_{i=0}^{N} r^{i} O_{i} \bmod L_{G}(\mathcal{M})
$$

for any $N \in \mathbb{N}$, with suitable $O_{i} \in L^{s}(\mathcal{M})$; in particular, $r^{i} O_{i}$ is flat of order $i$, with $i=0, \ldots, N$, in the pseudo-differential algebra. This statement is trivial for the subclass of differential operators where the expansion can be easily obtained from a Taylor expansion of the coefficients. We refer to ref. 46 concerning the extension of this concept to the whole class of pseudo-differential operators.

The recurrence scheme is based on the asymptotic expansion of the shifted Hamiltonian and its parametrix.

$$
\begin{aligned}
& \left(\sum_{i=0}^{N} r^{i} P_{i}\right)\left(\sum_{i=0}^{N} r^{i} A_{i}\right) \sim I \bmod L_{G}(\mathcal{M}), \\
& \left(\sum_{i=0}^{N} r^{i} A_{i}\right)\left(\sum_{i=0}^{N} r^{i} P_{i}\right) \sim I \bmod L_{G}(\mathcal{M})
\end{aligned}
$$

As already mentioned before, differential operators $A_{i}$ can be easily obtained via Taylor expansions. In an initial step, we construct $P_{0}$ from the zero'th order equation

$$
P_{0} A_{0}=I \bmod L_{G}(\mathcal{M}) \text {. }
$$

With this term at hand $P_{1}$ can be obtained from the first order recursion equation

$$
P_{0} r^{1} A_{1}+r^{1} P_{1} A_{0}=0 \bmod L_{G}(\mathcal{M}) .
$$

In general $O \in L^{s}(\mathcal{M})$ satisfies a commutator relation

$$
O r^{\beta}-r^{\beta} O_{\beta}=0 \bmod L_{G}(\mathcal{M}) \text { with } O_{\beta} \in L^{s}(\mathcal{M}) .
$$

With this relation and $P_{0}$ applied from the right, we get

$$
\begin{aligned}
r^{1} P_{1} & =-P_{0} r^{1} A_{1} P_{0} \bmod L_{G}(\mathcal{M}) \\
& =-r^{1} P_{0,1} A_{1} P_{0} \bmod L_{G}(\mathcal{M}) .
\end{aligned}
$$

which gives an explicit expression for $P_{1}$ in terms of known quantities. In principal, the recurrence scheme can be extended to arbitrarily high order, e.g. in second order, one gets the equation

$$
r^{1} P_{1} r^{1} A_{1}+r^{2} P_{2} A_{0}+P_{0} r^{2} A_{2}=0 \bmod L_{G}(\mathcal{M}),
$$

which becomes

$$
\begin{aligned}
r^{2} P_{2} & =-r^{1} P_{1} r^{1} A_{1} P_{0}-P_{0} r^{2} A_{2} P_{0} \bmod L_{G}(\mathcal{M}) \\
& =r^{2}\left(P_{0,1,1} A_{1,1} P_{0,1} A_{1} P_{0}-P_{0,2} A_{2} P_{0}\right) \bmod L_{G}(\mathcal{M}) .
\end{aligned}
$$

In order to extract asymptotic information it is essential to keep track of all Green operators which have been accumulated in the course of the recurrence scheme. 


\section{Parametrix and Green operator of the helium atom}

After a brief and fairly dense discussion of basic prerequisites and some necessary mathematical backgound of our approach in the previous section, we are ready to state our main result and discuss some of its implications. The recurrence scheme, outlined in Section 2.4, provides a constructive approach in order to derive explicit asymptotic expressions for parametrices and corresponding Green operators. An illustrative example for such a construction is the hydrogen atom which has been studied in ref. 47 .

The helium atom can be considered as a paradigm for electron correlation, in particular of electron-pairs, and provides the setting for the study of effective electron-pair models, which will be the subject of the following section. Therefore it represents a natural starting point for the asymptotic analysis of electron correlation. According to our previous discussion, it is the main outcome of our approach to derive an explizit expression for the Green operator, which encodes essential asymptotic information, $c f$. eqn (2.24) and (2.25). In the following, we want to discuss the Green operator corresponding to a shifted Hamiltonian of the helium atom (2.13), and extract some well known asymptotic properties, commonly known as Kato's cusp condition, from it. The technical details of the corresponding calculations are rather involved and therefore we refrain here from a detailed exposition. Instead, the interested reader is referred to a forthcoming publication, ${ }^{48}$ which contains all the relevant calculations and proofs in full length.

For the helium atom, the Green operator $G_{1}$ near an $e-e$ or $e-n$ edge has a leading order asymptotic expansion of the form

$$
\begin{aligned}
& G_{1} u\left(r, \varphi_{1}, \theta_{1}, y\right) \\
& \sim 2 t^{2}\left[\left(1+r t Z_{1}+r^{2}\left(-2+\frac{1}{3}\left(t Z_{1}\right)^{2}+\frac{1}{3} t Z_{2}\right)\right) \mathcal{P}_{0} \mathcal{Q}_{0,1}(u)(y)\right. \\
& +\frac{1}{6} r^{2} \mathcal{P}_{0} \mathcal{Q}_{0,2}(u)(y)+\left(\frac{1}{3} r+\frac{1}{6} t Z_{1} r^{2}\right) \mathcal{P}_{1} \mathcal{Q}_{1,1}(u)(y) \\
& \left.+\frac{1}{5} r^{2} \mathcal{P}_{2} \mathcal{Q}_{2,1}(u)(y)-\frac{1}{30} r^{2} \mathcal{P}_{2} \mathcal{Q}_{2,2}(u)(y)\right]+\mathcal{O}\left(r^{3}\right)
\end{aligned}
$$

with parameters

$$
\begin{aligned}
& Z_{1}:= \begin{cases}\frac{1}{\sqrt{2}} & \text { for } v_{e-e} \\
-Z & \text { for } v_{e-n}\end{cases} \\
& Z_{2}:=-t \lambda+ \begin{cases}-2 \sqrt{2} Z & \text { for } v_{e-e} \\
1-Z & \text { for } v_{e-n}\end{cases}
\end{aligned}
$$

where $\lambda$ might correspond to an eigenvalue and $\mathcal{P}_{l}, l=0,1$, $2, \ldots$, denote projection operators on subspaces which belong to eigenvalues $-l(l+1)$ of the Laplace-Beltrami operator on $S^{2}$, i.e. relative angular momenta of the electron-pair ( $e-e$ edge) or of an electron with respect to a nucleus ( $e-n$ edge). The linear operators $\mathcal{Q}_{l, j}$ map $u\left(r, \varphi_{1}, \theta_{1}, t, \varphi_{2}, \theta_{2}\right)$ into a function $\mathcal{Q}_{l, j}(u)\left(\varphi_{1}, \theta_{1}, t, \varphi_{2}, \theta_{2}\right)$ which does not depend on $r$ anymore. Explicit expressions of the operators $\mathcal{Q}_{l, j}$ are rather involved and will be given in a forthcoming publication. ${ }^{48}$ By the projection operators $\mathcal{P}_{l}, l=0,1,2, \ldots$, the function $\mathcal{Q}_{l, j}(u)$ is furthermore projected with respect to the angular variables $\theta_{1}, \varphi_{1}$ on subspaces of relative angular momentum eigenvalues, i.e.,

$$
\mathcal{P}_{l} \mathcal{Q}_{l, j}(u)\left(\varphi_{1}, \theta_{1}, t, \varphi_{2}, \theta_{2}\right)=\sum_{m=-l}^{l} Y_{l m}\left(\theta_{1}, \varphi_{1}\right) v_{l m j}\left(t, \theta_{2}, \varphi_{2}\right)
$$

with

$$
v_{l m j}\left(t, \theta_{2}, \varphi_{2}\right):=\int_{S^{2}} \bar{Y}_{l, m}\left(\theta_{1}, \varphi_{1}\right) \mathcal{Q}_{l, j}(u)\left(\varphi_{1}, \theta_{1}, t, \varphi_{2}, \theta_{2}\right) \mathrm{d} \theta_{1} \mathrm{~d} \varphi_{1},
$$

where $Y_{l m},-l \leq m \leq l$, denote the corresponding spherical harmonics.

At this point it seems to be appropriate to discuss our main result (3.1) in less technical terms and to clarify its relation to previous work, in particular with respect to various higher-order cusp conditions mentioned in the quantum chemistry literature. First of all, (3.1) together with the corresponding parametrix represents an approximate inverse of a shifted Hamiltonian (2.13), here considered as an operator acting between appropriate function spaces, where the shift parameter might be an eigenvalue or not. In conventional quantum chemistry, only the inverse of a shifted noninteracting Hamiltonian, acting on the virtual part of Hilbert space, seems to be a valid option and provides $e$.g. the basis of the CC iteration scheme. It is a subject of our future work to study possible local modifications of standard iteration schemes using interacting Green operators and parametrices in order to improve convergence near the $e-e$ edge. This seems to be possible, because the Green operator (3.1) maps any function $u$ into a function with this specific asymptotic behaviour.

In the case of a linear electron-pair model of the general type (2.23), it is possible to determine the asymptotic behaviour of its solution from eqn (2.24) or (2.25) using the corresponding asymptotic Green operator and parametrix. It should be mentioned, that the electron-pair models discussed below require only trivial modifications of the Green operator (3.1) and corresponding parametrix. Once again, we want to emphasize that the asymptotic recurrence scheme of Section 2.4 is constructive and can in principle be extended to any order in the $e-e$ distance. Therefore it is possible to calculate the asymptotic expansion (3.1) to any order in a systematic way and determine the asymptotic behaviour well beyond Kato's cusp condition. Here we want to mentioned that various generalizations of Kato's cusp condition have been already discussed in the literature, $c f$. ref. 49-52. Let us point to an important difference with respect to the present work. It is a common assumption in these papers that the most general underlying asymptotic expansion of a wavefunction is of the general form

$$
u(r, \theta, \phi, \mathbf{s}) \sim \sum_{0 \leq l} \sum_{0 \leq n} r^{l+n} \sum_{m=-l}^{l} u_{l m n}(\mathbf{s}) Y_{l m}(\theta, \phi),
$$


where $r, \theta, \phi$ denotes spherical coordinates with respect to the $e-e$ distance and $\mathbf{s}$ refers to the center of mass of an electron pair. However, an asymptotic expansion of the form (3.5) cannot be taken for granted. It might happen, e.g., that logarithmic terms like $r^{k} \ln r$ show up. Actually, logarithmic terms are well known from the Fock expansion of the helium atom near the corner singularity where both electrons approach the nucleus. The absence of logarithms and noninteger exponents in the asymptotic expansion at the $e-e$ edge requires a proof. Only recently, Fournais et al. ${ }^{19}$ proved rigorously the existence of an asymptotic expansion (3.5) near an $e-e$ edge for eigenfunctions of many-electron Hamiltonians. Their proof is rather sophisticated and should be considered as a subsequent justification of the work done in quantum chemistry. Because it restricts to eigenfunctions, ref. 19 applies to (2.23) in the case $f=0$ and $\lambda$ an eigenvalue, but not for general right hand sides $f$ and arbitrary values of the shift parameter $\lambda$, which is of relevance for approximate linear many-particle models, to be discussed below. In contrast to this, our approach does not make any a priori assumptions concerning the asymptotic behaviour. It only requires that the right hand side $f$ has an asymptotic expansion of the general type (2.21), $c f$. ref. 20. Therefore, integer exponents and the absence of logarithms in the asymptotic expansion of the Green operator (3.1) are an outcome of our calculation and not the consequence of any assumption. In particular, this is of significance for approximate models, derived from CC theory, where the proof of ref. 19 does not immediately apply.

\subsection{Kato's cusp condition revisited}

With the asymptotic Green operator at hand, it is straightforward to derive Kato's cusp condition ${ }^{14}$ from the leading order terms. Let us first consider the asymptotic behaviour of wavefunctions near the $e-e$ edge. With respect to the euclidean distance between both electrons

$$
x_{12}:=\left|\mathbf{x}_{1}-\mathbf{x}_{2}\right|=\sqrt{2} t \sin r=\sqrt{2} t\left(r+\mathcal{O}\left(r^{3}\right)\right),
$$

the asymptotic expansion for a symmetric (singlet) state $\Psi_{\mathrm{s}}$ of the electron-pair

$$
G_{l} \Psi_{\mathrm{s}} \sim\left(1+\frac{1}{2} x_{12}\right) \frac{1}{\sqrt{\pi}} t^{2} v_{001}\left(t, \theta_{2}, \varphi_{2}\right)+\mathcal{O}\left(x_{12}{ }^{2}\right),
$$

is perfectly equivalent to Kato's "cusp" condition, $\mathbf{x}_{2} \neq 0$,

$$
\left.\frac{1}{4 \pi} \frac{\partial \int_{S^{2}} \Psi_{\mathrm{s}}\left(x_{12}, \omega_{12}, \mathbf{x}_{2}\right) \mathrm{d} \omega_{12}}{\partial x_{12}}\right|_{x_{12}=0}=\frac{1}{2} \Psi_{\mathrm{s}}\left(0, \cdot, \mathbf{x}_{2}\right),
$$

where $\omega_{12}$ denotes the corresponding angular coordinates of the $e-e$ distance. For an antisymmetric (triplet) state $\Psi_{\mathrm{t}}$ of the electron-pair, we get

$$
\begin{gathered}
G_{l} \Psi_{\mathrm{t}} \sim \sqrt{2} \operatorname{tr}\left(1+\frac{1}{4} \sqrt{2} t r\right) \sum_{m=-1}^{1} Y_{1 m}\left(\theta_{1}, \varphi_{1}\right) \frac{\sqrt{2}}{3} t v_{1 m j}\left(t, \theta_{2}, \varphi_{2}\right) \\
\sim x_{12}\left(1+\frac{1}{4} x_{12}\right) \sum_{m=-1}^{1} Y_{l m}\left(\theta_{1}, \varphi_{1}\right) \frac{\sqrt{2}}{3} t v_{1 m j}\left(t, \theta_{2}, \varphi_{2}\right)+\cdots
\end{gathered}
$$

At next, we want to discuss the asymptotic behaviour of wavefunctions near an $e-n$ edge. Let us first consider states with $\mathcal{P}_{0} \Psi \neq 0$, the asymptotic expansion for such states yields

$$
G_{l} \Psi \sim\left(1-Z\left|\mathbf{x}_{1}\right|\right) \frac{1}{\sqrt{\pi}} t^{2} v_{001}\left(t, \theta_{2}, \varphi_{2}\right)+\mathcal{O}\left(\left|\mathbf{x}_{1}\right|^{2}\right),
$$

with respect to the euclidean distance between an electron and a nucleus. Again this is perfectly equivalent to Kato's cusp condition, $x_{1}:=\left|\mathbf{x}_{1}\right|$ and $\mathbf{x}_{2} \neq 0$,

$$
\left.\frac{1}{4 \pi} \frac{\partial \int_{S^{2}} \Psi\left(x_{1}, \omega_{1}, \mathbf{x}_{2}\right) \mathrm{d} \omega_{1}}{\partial x_{1}}\right|_{x_{1}=0}=-Z \Psi\left(0, \cdot, \mathbf{x}_{2}\right),
$$

where $\omega_{1}$ denotes the corresponding angular coordinates of the $e-n$ distance. For states with $\mathcal{P}_{0} \Psi=0$ and $\mathcal{P}_{0} \Psi \neq 0$, e.g. highly excited Rydberg states, the asymptotic expansion

$$
G_{l} \Psi \sim\left|\mathbf{x}_{1}\right|\left(1-\frac{1}{2} Z\left|\mathbf{x}_{1}\right|\right) \sum_{m=-1}^{1} Y_{l m}\left(\theta_{1}, \varphi_{1}\right) \frac{2}{3} t v_{1 m j}\left(t, \theta_{2}, \varphi_{2}\right) .
$$

resembles to the $2 \mathrm{p}$ state of a $\mathrm{He}^{+}$ion.

\section{Effective electron-pair models derived from $\mathrm{CC}$ theory}

In quantum chemistry a multitude of electron-pair models are known, among the most popular are CCSD and various variants of CEPA. It is not our intention to make extensive comments on these models or to discuss how these models are related to each other. CC theory, however, seems to provide a unified framework, $c f .{ }^{53}$ and is a good starting point to establish a hierarchy among these models. The models are based on an effective single particle model, like Hartree-Fock, and assign to each pair of occupied orbitals a wavefunction $\Psi_{i, j}^{(2)}$ which represents an electron-pair embedded in an effective mean-field generated by the remaining electrons. Let us assume the canonical decomposition of the effective electron-pair wavefunction

$$
\Psi_{i, j}^{(2)}\left(\underline{\mathbf{x}}_{1}, \underline{\mathbf{x}}_{2}\right)=\Psi_{i, j}^{(1)}\left(\underline{\mathbf{x}}_{1}, \underline{\mathbf{x}}_{2}\right)+\tau_{i, j}\left(\underline{\mathbf{x}}_{1}, \underline{\mathbf{x}}_{2}\right)
$$

into a noninteracting part $\Psi_{i, j}^{(1)}$ and the pair-amplitude $\tau_{i, j}$. In any effective electron-pair model, pair-amplitudes rely on the constraint

$$
\mathfrak{Q} \tau_{i, j}\left(\underline{\mathbf{x}}_{1}, \underline{\mathbf{x}}_{2}\right)=\tau_{i, j}\left(\underline{\mathbf{x}}_{1}, \underline{\mathbf{x}}_{2}\right),
$$

imposed by the projection operator, commonly known as strong orthogonality operator ${ }^{55}$

$$
\mathfrak{Q}:=\left(1-\mathfrak{q}_{1}\right)\left(1-\mathfrak{q}_{2}\right) \text { with } \mathfrak{q}:=\sum_{i=1}^{N}\left|\phi_{i}\right\rangle\left\langle\phi_{i}\right|,
$$

where $\phi_{i}$, with $i=1,2, \ldots, N$, represent occupied orbitals. The physical reason behind is Pauli's principle which excludes the subspace assigned to the remaining $N-2$ particles from the Hilbert space of the pair and an orthogonality constraint between the two parts $\Psi_{i, j}^{(1)}$ and $\tau_{i, j}$ of the pair wavefunction.

In the present work our focus is on the behaviour of wavefunctions near the $e-e$ edge. It is well known from quantum 
many-body theory, that the so-called ladder diagrams give the dominant contribution to short-range correlation. A simple model where ladder diagrams are summed to infinite order is the Bethe-Goldstone (BG) equation, which can be easily derived from CCSD by neglecting all interactions among different electron-pairs. The BG equation can be written in the following form

$$
\begin{aligned}
& \mathfrak{Q}\left(-\frac{1}{2} \Delta_{1}+\mathfrak{v}_{1}-\frac{1}{2} \Delta_{2}+\mathfrak{v}_{2}-E_{i, j}\right) \tau_{i, j}\left(\underline{\mathbf{x}}_{1}, \underline{\mathbf{x}}_{2}\right) \\
& \quad=-\mathfrak{Q} \frac{1}{\left|\mathbf{x}_{1}-\mathbf{x}_{2}\right|} \Psi_{i, j}^{(1)}\left(\underline{\mathbf{x}}_{1}, \underline{\mathbf{x}}_{2}\right)-\mathfrak{Q} V_{\text {fluc }}^{(i j)}\left(\underline{\mathbf{x}}_{1}, \underline{\mathbf{x}}_{2}\right) \tau_{i, j}\left(\underline{\mathbf{x}}_{1}, \underline{\mathbf{x}}_{2}\right),
\end{aligned}
$$

with coefficient matrix

$$
\begin{aligned}
E_{i j}:= & \left\langle\phi_{i},\left(-\frac{1}{2} \Delta+\mathfrak{v}\right) \phi_{i}\right\rangle+\left\langle\phi_{j},\left(-\frac{1}{2} \Delta+\mathfrak{v}\right) \phi_{j}\right\rangle \\
& +\frac{1}{2}\left\langle\Psi_{i, j}^{(1)}, \frac{1}{\left|\mathbf{x}_{1}-\mathbf{x}_{2}\right|} \tau_{i, j}\right\rangle,
\end{aligned}
$$

and fluctuation potential

$$
\begin{aligned}
V_{\text {fluc }}^{(i j)}\left(\underline{\mathbf{x}}_{1}, \underline{\mathbf{x}}_{2}\right):= & \frac{1}{\left|\mathbf{x}_{1}-\mathbf{x}_{2}\right|}-\mathfrak{v}_{H x}^{(i, j)}\left(\underline{\mathbf{x}}_{1}\right)-\mathfrak{v}_{H x}^{(i, j)}\left(\underline{\mathbf{x}}_{2}\right) \\
& +\frac{1}{2}\left\langle\Psi_{i, j}^{(1)}, \frac{1}{\left|\mathbf{x}_{1}-\mathbf{x}_{2}\right|} \Psi_{i, j}^{(1)}\right\rangle
\end{aligned}
$$

in which

$$
\mathfrak{v}_{H x}^{(i, j)}:=\mathfrak{v}_{H}^{(i)}+\mathfrak{v}_{H}^{(j)}+\mathfrak{v}_{x}^{(i)}+\mathfrak{v}_{x}^{(j)}
$$

represents the contribution of orbitals $i, j$ to the Hartree and exchange potential, respectively. The BG eqn (4.4) is still nonlinear due to the last term of the coefficient matrix $E_{i j}$ which depends on $\tau_{i, j}$. In the following, we skip this term and consider a simplified linear BG equation with coefficient matrix

$$
E_{i j}^{(0)}:=\left\langle\phi_{i},\left(-\frac{1}{2} \Delta+\mathfrak{v}\right) \phi_{i}\right\rangle+\left\langle\phi_{j},\left(-\frac{1}{2} \Delta+\mathfrak{v}\right) \phi_{j}\right\rangle=\varepsilon_{i}+\varepsilon_{j} .
$$

The BG equation can be further simplified by neglecting all second order terms. This yields the equation for RayleighSchrödinger (RS) 1'st order perturbation theory

$$
\begin{aligned}
& \left(-\frac{1}{2} \Delta_{1}+\mathfrak{v}_{1}-\frac{1}{2} \Delta_{2}+\mathfrak{v}_{2}-\varepsilon_{i}-\varepsilon_{j}\right) \tau_{i, j}\left(\underline{\mathbf{x}}_{1}, \underline{\mathbf{x}}_{2}\right) \\
& =-\mathfrak{Q} \frac{1}{\left|\mathbf{x}_{1}-\mathbf{x}_{2}\right|} \Psi_{i, j}^{(1)}\left(\underline{\mathbf{x}}_{1}, \underline{\mathbf{x}}_{2}\right),
\end{aligned}
$$

which provides the starting point for an iterative solution of the BG equation.

\subsection{Kato's cusp condition for effective electron-pair models}

The BG and 1'st order RS models are linear equations of the general form (2.23), where the asymptotic behaviour can be obtained from the parametrix and Green operator according to eqn (2.25). An explicit calculation yields

$$
\left.\mathcal{P}_{0} \tau_{i, j} \sim \tau_{i, j}\right|_{r=0}+\left.\frac{1}{2}(\sqrt{2} r t)\left(\Psi_{i, j}^{(1)}+\tau_{i, j}\right)\right|_{r=0}+\mathcal{O}\left(r^{2}\right)
$$

or the equivalent expression

$$
\left.\mathcal{P}_{0} \Psi_{i, j}^{(2)} \sim \Psi_{i, j}^{(2)}\right|_{r=0}+\left.\frac{1}{2}(\sqrt{2} r t) \Psi_{i, j}^{(2)}\right|_{r=0}+\mathcal{O}\left(r^{2}\right) .
$$

The latter in turn is equivalent to Kato's cusp condition

$$
\left.\partial_{x_{12}} \mathcal{P}_{0} \Psi_{i, j}^{(2)}\right|_{r=0}=\left.\frac{1}{2} \Psi_{i, j}^{(2)}\right|_{r=0} .
$$

For comparison, 1'st order RS perturbation theory yields

$$
\left.\mathcal{P}_{0} \Psi_{i, j}^{(2)} \sim \Psi_{i, j}^{(2)}\right|_{r=0}+\left.\frac{1}{2}(\sqrt{2} r t) \Psi_{i, j}^{(1)}\right|_{r=0}+\mathcal{O}\left(r^{2}\right)
$$

or equivalently

$$
\left.\partial_{x_{12}} \mathcal{P}_{0} \Psi_{i, j}^{(2)}\right|_{r=0}=\left.\frac{1}{2} \Psi_{i, j}^{(1)}\right|_{r=0}
$$

which can be considered as a 1'st order approximation of Kato's cusp condition, $c f$. the discussion in ref. 54 .

\subsection{Asymptotic singular analysis of iteration schemes}

Usually, the BG equation is solved via the fixed-point iteration scheme

$$
\left(-\frac{1}{2} \Delta_{1}+\mathfrak{v}_{1}-\frac{1}{2} \Delta_{2}+\mathfrak{v}_{2}-E_{i, j}^{(0)}\right) \tau_{i, j}^{(n+1)}\left(\underline{\mathbf{x}}_{1}, \underline{\mathbf{x}}_{2}\right)
$$

$$
=-\mathfrak{Q} \frac{1}{\left|\mathbf{x}_{1}-\mathbf{x}_{2}\right|} \Psi_{i, j}^{(1)}\left(\underline{\mathbf{x}}_{1}, \underline{\mathbf{x}}_{2}\right)-\mathfrak{Q} V_{\text {fluc }}^{(i, j)}\left(\mathbf{x}_{1}, \mathbf{x}_{2}\right) \tau_{i, j}^{(n)}\left(\underline{\mathbf{x}}_{1}, \underline{\mathbf{x}}_{2}\right)
$$

with initial guess $\tau_{i, j}^{(0)}=0$, i.e., starting from first-order RS perturbation theory. The corresponding diagrammatic representation of (4.15), via Goldstone ladder diagrams, is shown in Fig. 5. In every single iteration step the asymptotic expansion becomes

$$
\left.\mathcal{P}_{0} \tau_{i, j}^{(n+1)} \sim \tau_{i, j}^{(n+1)}\right|_{r=0}+\left.\frac{1}{2}(\sqrt{2} r t)\left(\Psi_{i, j}^{(1)}+\tau_{i, j}^{(n)}\right)\right|_{r=0}+\mathcal{O}\left(r^{2}\right)
$$

From this it can be seen, how the fixed-point iteration scheme eventually converges towards Kato's cusp condition. Let us define

$$
\begin{gathered}
\Delta \tau_{i, j}^{(1)}:=\tau_{i, j}^{(1)} \\
\Delta \tau_{i, j}^{(2)}:=\tau_{i, j}^{(2)}-\tau_{i, j}^{(1)} \\
\vdots \\
\Delta \tau_{i, j}^{(n)}:=\tau_{i, j}^{(n)}-\tau_{i, j}^{(n-1)},
\end{gathered}
$$

i.e., $\Delta \tau_{i, j}^{(n)}$ corresponds to the contribution of the $n$ 'th order ladder diagram, $c f$. Fig. 5, and the asymptotic expansion of such diagrams for $n>1$ is given by

$$
\left.\mathcal{P}_{0} \Delta \tau_{i, j}^{(n)} \sim \Delta \tau_{i, j}^{(n)}\right|_{r=0}+\left.\frac{1}{2}(\sqrt{2} r t) \Delta \tau_{i, j}^{(n-1)}\right|_{r=0}+\mathcal{O}\left(r^{2}\right) .
$$



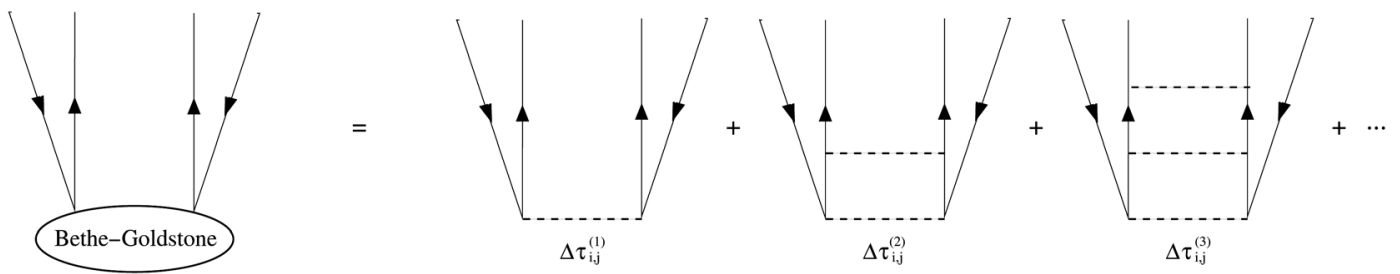

Fig. 5 Iterative solution of the BG equation.

This behaviour can be subsumed by the following formal perturbation scheme. Let us take

$$
\Psi_{i, j}(\lambda)=\Psi_{i, j}^{(1)}+\sum_{0 \leq n} \lambda^{n} \Delta \tau_{i, j}^{(n)},
$$

with (4.18), the cusp condition becomes

$$
\left.\partial_{x_{12}} \mathcal{P}_{0} \Psi_{i, j}(\lambda)\right|_{r=0}=\left.\frac{1}{2} \lambda \Psi_{i, j}(\lambda)\right|_{r=0} .
$$

Formal perturbation theory yields

$$
\begin{gathered}
\left.\partial_{x_{12}} \mathcal{P}_{0} \Psi_{i, j}^{(1)}\right|_{r=0}=0 \\
\left.\partial_{x_{12}} \mathcal{P}_{0} \Delta \tau_{i, j}^{(1)}\right|_{r=0}=\left.\frac{1}{2} \Psi_{i, j}^{(1)}\right|_{r=0} \\
\left.\partial_{x_{12}} \mathcal{P}_{0} \Delta \tau_{i, j}^{(2)}\right|_{r=0}=\left.\frac{1}{2} \Delta \tau_{i, j}^{(1)}\right|_{r=0}
\end{gathered}
$$

which means that (4.20) reproduces order by order our asymptotic result.

\subsection{Beyond ladder diagrams}

The BG equation represents a rather crude approximation and in order to deal with state of the art models like CCSD or CEPA, it is essential to take further classes of diagrams into account. In particular it becomes necessary to consider nonlinear couplings between pair amplitudes, like in ring diagrams. This is the subject of our present work where we want to study the effect of various classes of diagrams on the asymptotic behaviour. It is rather obvious from our analysis that such diagrams mainly affect higher-order terms in the asymptotic expansion of pair amplitudes. Nevertheless, it seems to be premature to make any precise statement. In order to get a rough idea which steps are required for nonlinear models, we want to refer to ref. 45, where an asymptotic singular analysis has been performed for the nonlinear Hartree-Fock model.

\section{Adaptive wavelet approximation of pair-amplitudes}

The asymptotic analysis of the pair-amplitudes permits a rigorous statement concerning their adaptive approximation by tensor product wavelet bases. Given a three dimensional isotropic wavelet basis $\left\{\gamma_{\alpha}, \alpha \in \Lambda\right\}$ with index set $\Lambda$, we consider the tensor product wavelet expansion of pair-amplitudes

$$
\tau_{i, j}\left(\mathbf{x}_{1}, \mathbf{x}_{2}\right)=\sum_{\alpha, \beta \in \Lambda} c_{\alpha \beta}\left(\gamma_{\alpha}\left(\mathbf{x}_{1}\right) \gamma_{\beta}\left(\mathbf{x}_{2}\right)+\gamma_{\beta}\left(\mathbf{x}_{1}\right) \gamma_{\alpha}\left(\mathbf{x}_{2}\right)\right) .
$$

For computer simulations one has to restrict the expansion to a finite number of wavelets. Obviously it is desirable to select wavelets in an adaptive manner according to their significance. The underlying mathematical concept is best $N$-term approximation which belongs to the realm of nonlinear approximation theory. For a detailed exposition of this subject we refer to ref. 56. Loosely speaking, we consider for a given basis, the best possible approximation of a function in the nonlinear subset $\Sigma_{N}$ which consists of all possible linear combinations of at most $N$ basis functions, i.e.,

$$
\Sigma_{N}:=\left\{\sum_{(\alpha, \beta) \in \Delta} c_{\alpha \beta}\left(\gamma_{\alpha} \otimes \gamma_{\beta}+\gamma_{\beta} \otimes \gamma_{\alpha}\right): \quad \Delta \subset \Lambda, \# \Delta \leq N\right\}
$$

Here, the approximation error

$$
\sigma_{N}(u):=\inf _{f_{N} \in \Sigma_{N}}\left\|u-u_{N}\right\|_{H}
$$

is given with respect to the norm of an appropriate Hilbert space $H$. A best $N$-term approximation space $A^{\alpha}(H)$ for a Hilbert space $H$ contains all functions for which the error has convergence rate $\sigma_{N}(u) \sim N^{-\alpha}$. Actually, the function spaces $A^{\alpha}(H)$ correspond to certain Besov spaces where wavelets provide stable Riesz bases. This property enables a direct estimate of the approximation error from wavelet coefficients.

In order to study $N$-term approximation rates for pairamplitudes, it is important to establish the following growth estimates

$$
\begin{gathered}
\left|\partial_{\boldsymbol{x}}^{\alpha} \partial_{\boldsymbol{y}}^{\beta} \tau_{i, j}\left(\boldsymbol{x}_{1}, \boldsymbol{x}_{2}\right)\right| \leq c_{\alpha, \beta}\left|\boldsymbol{x}_{1}-\boldsymbol{x}_{2}\right|^{1-|\alpha|-|\beta|}, \text { for } \boldsymbol{x}_{1} \neq \boldsymbol{x}_{2} \\
\text { and }|\alpha|+|\beta| \geq 1,
\end{gathered}
$$

with respect to their partial derivatives near the $e-e$ edge. Such estimates are a simple corollary of our asymptotic analysis. It is an immediate consequence of previous work, $c f .,{ }^{57}$ that with respect to the Sobolev space $H^{1}$ the following error estimate

$$
\left\|\tau-\tau_{N}\right\|_{H^{1}} \leq C N^{-\frac{1}{2}+\varepsilon}
$$

for any $\varepsilon>0$, can be achieved. Therefore, the error in energy converges with $O\left(N^{-1+\varepsilon}\right)$, for any $\varepsilon>0$, which might be compared with the empirical convergence rate of $O\left(N^{-\frac{1}{2}}\right)$ for
correlation consistent basis sets given in ref. 58 . 


\section{Conclusions}

We presented a general approach to study the asymptotic behaviour of wavefunctions near coalescence points of particles. It can be applied to the original many-electron Schrödinger equation, as well as to approximate models in the realm of CC theory. Singular analysis provides the abstract mathematical framework and it was our task to show that electronic structure theory actually fits into it. In this respect, we followed a top down approach, with an abstract mathematical theory at the beginning and explicit calculations of asymptotic parametrices and Green operators at the end. Much remains to be done, in particular the extension of our analysis to higher order terms and the asymptotic analysis of nonlinear couplings between pair-amplitudes, which appear in CC theory. Another important aspect of our present work is the incorporation of asymptotic parametrices and Green operators into numerical methods using systematic bases like wavelets.

\section{References}

1 H. Yserentant, Numer. Math., 2004, 98, 731-759; H. Yserentant, Numer. Math., 2005, 101, 381-389.

2 H. Yserentant, Regularity and Approximability of Electronic Wave Functions, Lecture Notes in Mathematics, Springer, 2010, vol. 2000.

3 H. Yserentant, ESAIM: Math. Modell. Numer. Anal., 2011, 45, 803-824.

4 A. Zeiser, Constr. Approx., 2012, 35, 1-30.

5 H.-C. Kreusler and H. Yserentant, Numer. Math., 2012, 121, 781-802.

6 M. Griebel and J. Hamaeckers, ESAIM: Math. Modell. Numer. Anal., 2007, 41, 215-247.

7 M. Griebel and J. Hamaeckers, Z. Phys. Chem., 2010, 224, 527-543.

8 M. Bachmayr, ESAIM: Math. Modell. Numer. Anal., 2012, 46, 1337-1362.

9 J. Paldus and X. Li, in Adv. Chem. Phys., ed. I. Prigogine and S. A. Rice, 1999, vol. 110, pp. 1-175.

10 R. J. Bartlett and M. Musial, Coupled-cluster theory in quantum chemistry, Rev. Mod. Phys., 2007, 79, 291-352.

11 R. Schneider, Numer. Math., 2009, 113, 433-471.

12 T. Rohwedder, ESAIM: Math. Modell. Numer. Anal., 2013, 47, 421-447.

13 T. Rohwedder and R. Schneider, ESAIM: Math. Modell. Numer. Anal., 2013, 47, 1553-1582.

14 T. Kato, Commun. Pure Appl. Math., 1957, 10, 151-177.

15 M. Hoffmann-Ostenhof and R. Seiler, Phys. Rev. A: At., Mol., Opt. Phys., 1981, 23, 21-23.

16 M. Hoffmann-Ostenhof and T. Hoffmann-Ostenhof, Commun. Partial Diff. Eq., 1992, 17, 491-522.

17 M. Hoffmann-Ostenhof, T. Hoffmann-Ostenhof and H. Stremnitzer, Commun. Math. Phys., 1994, 163, 185-215.

18 S. Fournais, M. Hoffmann-Ostenhof, T. Hoffmann-Ostenhof and T. Østergaard Sørensen, Commun. Math. Phys., 2005, 255, 183-227.
19 S. Fournais, M. Hoffmann-Ostenhof, T. Hoffmann-Ostenhof and T. Østergaard Sørensen, Commun. Math. Phys., 2009, 289, 291-310.

20 Y. V. Egorov and B.-W. Schulze, Pseudo-Differential Operators, Singularities, Applications, Birkhäuser, 1997.

21 B.-W. Schulze, Boundary Value Problems and Singular PseudoDifferential Operators, Wiley, 1998.

22 G. Harutyunyan and B.-W. Schulze, Elliptic Mixed, Transmission and Singular Crack Problems, EMS Tracts in Mathematics, European Math. Soc., 2008, vol. 4.

23 K. Akutagawa, G. Carron and R. Mazzeo, preprint 2014, arXiv:1409.0154v1.

24 E. Hunsicker, V. Nistor and J. O. Sofo, J. Math. Phys., 2008, 49, 083501.

25 B. Ammann, C. Carvalho and V. Nistor, Lett. Math. Phys., 2012, 101, 49-98.

26 R. Thom, Bull. Amer. Math. Soc., 1969, 75, 240-284.

27 J. Mather, Notes on topological stability, Harvard University, 1970.

28 J. Leray, Actes du 6ème Congres du Groupement des Mathematiciens d'Expression Latine, Gauthier-Villars, 1982, pp. 179-187.

29 J. Leray, Lect. Notes Phys., Springer, 1984, vol. 195, pp. 235-247.

$30 \mathrm{~J}$. Leray, Methods of functional analysis and theory of elliptic operators: Naples 1982, Universita di Napoli, 1982.

31 J. D. Morgan III, Theor. Chim. Acta, 1986, 69, 181-223.

32 W. Kutzelnigg, Theor. Chim. Acta, 1985, 68, 445-469.

33 V. A. Fock, in V. A. Fock - Selected Works: Quantum Mechanics and Quantum Field Theory, ed. L. D. Faddeev, L. A. Khalfin and I. V. Komarov, Chapman \& Hall/CRC, 2004, pp. 525-538.

34 P. C. Abbott and E. N. Maslen, J. Phys. A: Math. Gen., 1987, 20, 2043-2075.

35 J. E. Gottschalk, P. C. Abbott and E. N. Maslen, J. Phys. A: Math. Gen., 1987, 20, 2077-2104.

36 J. E. Gottschalk and E. N. Maslen, J. Phys. A: Math. Gen., 1987, 20, 2781-2803.

37 E. A. Hylleraas, Z. Phys., 1929, 54, 347-366.

38 C. L. Pekeris, Phys. Rev., 1958, 112, 1649-1658.

39 K. Frankowski and C. L. Pekeris, Phys. Rev., 1966, 146, 46-49.

40 D. E. Freund, B. D. Huxtable and J. D. Morgan III, Phys. Rev. A: At., Mol., Opt. Phys., 1984, 29, 980-982.

41 P. M. Morse and H. Feshbach, Methods of Theoretical Physics, McGraw-Hill, 1953.

42 N. F. Mott and H. S. W. Massey, The Theory of Atomic Collisions, Oxford University Press, 3rd edn, 1965.

43 K. D. Granzow, J. Math. Phys., 1963, 4, 897-900.

44 H.-J. Flad and G. Harutyunyan, Proc. AIMS Conference on Dynamical Systems, Differential Equations and Applications, Dresden, 2010.

45 H.-J. Flad, R. Schneider and B.-W. Schulze, Math. Methods Appl. Sci., 2008, 31, 2172-2201.

46 H.-J. Flad, G. Harutyunyan, B.-W. Schulze, preprint 2010, arXiv:1010.1453v2.

47 H.-J. Flad, G. Harutyunyan, R. Schneider and B.-W. Schulze, manuscripta math., 2011, 135, 497-519.

48 H.-J. Flad, G. Harutyunyan and B.-W. Schulze, in preparation, preprint 2015. 
49 V. A. Rassolov and D. M. Chipman, J. Chem. Phys., 1996, 104, 9908-9912.

50 D. P. Tew, J. Chem. Phys., 2008, 129, 014104.

51 Y. I. Kurokawa, H. Nakashima and H. Nakatsuji, J. Chem. Phys., 2013, 139, 044114.

52 Y. I. Kurokawa, H. Nakashima and H. Nakatsuji, J. Chem. Phys., 2014, 140, 214103.

53 W. Kutzelnigg, in Methods of Electronic Structure Theory, Modern Theoretical Chemistry, Modern Theoretical Chemistry, ed. H. F. Schaefer III, Plenum, 1977, vol. 3, pp. 129-188.
54 C. Hättig, W. Klopper, A. Köhn and D. P. Tew, Chem. Rev., 2012, 112, 4-74.

55 K. Szalewicz, B. Jeziorski, H. J. Monkhorst and J. G. Zabolitzky, J. Chem. Phys., 1983, 78, 1420-1430.

56 R. A. DeVore, Acta Numerica, 1998, 7, 51-150.

57 H.-J. Flad, W. Hackbusch and R. Schneider, ESAIM: Math. Modell. Numer. Anal., 2007, 41, 261-279.

58 A. Halkier, T. Helgaker, P. Jørgensen, W. Klopper, H. Koch, J. Olsen and A. K. Wilson, Chem. Phys. Lett., 1998, 286, 243-252. 